\title{
Co-occurrence of DSM-IV mental disorders and alcohol use disorder among adult Chinese males
}

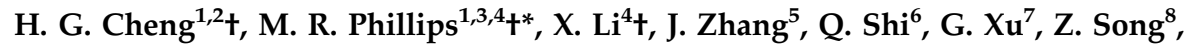 \\ Z. Ding ${ }^{9}$ and S. Pang ${ }^{10}$ \\ ${ }^{1}$ Shanghai Mental Health Center, Shanghai Jiao Tong University School of Medicine, China \\ ${ }^{2}$ Department of Epidemiology, Michigan State University, East Lansing, MI, USA \\ ${ }^{3}$ Departments of Psychiatry and Global Health, Emory University, USA \\ ${ }^{4}$ WHO Collaborating Center for Research and Training in Suicide Prevention, Beijing Hui Long Guan Hospital, Beijing, China \\ ${ }^{5}$ Shandong Provincial Mental Health Center, Jinan City, Shandong Province, China \\ ${ }^{6}$ Tong De Hospital of Zhejiang Province, Hangzhou City, Zhejiang Province, China \\ ${ }^{7}$ Tianjin Mental Health Center, Tianjin, China \\ ${ }^{8}$ The 3rd People's Hospital of Qinghai Province, Xining City, Qinghai Province, China \\ ${ }^{9}$ Tianshui City Mental Hospital, Tianshui City, Gansu Province, China \\ ${ }^{10}$ Qingdao Mental Health Centre, Qingdao City, Shandong Province, China
}

Background. Studies from high-income countries report moderate-to-strong positive associations between alcohol use disorder (AUD) and other mental disorders, but there is little evidence about the comorbidity of AUD from low-andmiddle-income countries.

Methods. A sample of 74752 adults from five provinces that account for $>12 \%$ of China's adult population was screened using the General Health Questionnaire, and the Structured Clinical Interview for DSM-IV was administered by psychiatrists to a subsample of 9619 males. The associations between AUD and other mental disorders at each site and the characteristics of men with AUD with and without comorbid mental disorders were estimated using logistic regression and summarized across sites using meta-analysis. Generalized estimation equations estimated the associations between the clinical features of alcohol dependence and comorbidity.

Results. Robust inverse associations were found between current AUD and any mood disorder (adjusted OR =0.6, 95\% $\mathrm{CI}=0.4-0.8)$ and any anxiety disorder $(\mathrm{OR}=0.5,95 \% \mathrm{CI}=0.3-1.0)$. Compared with men without AUD, men with AUD without comorbid disorders were more likely to be middle-aged, to be currently married, and to have higher family incomes. Men with comorbid AUD and other disorders were more likely to have the clinical features of alcohol dependence than men with AUD without comorbid disorders.

Conclusions. Inverse associations between AUD and other mental disorders and the higher social status of men with AUD than men without AUD found in this large, representative sample of community-dwelling Chinese males highlight the importance of considering the local substance-use culture when designing clinical or preventive interventions for addictive conditions.

Received 26 February 2017; Revised 20 April 2017; Accepted 24 April 2017; First published online 29 May 2017

Key words: Alcohol use disorder, China, community-based, comorbidity.

\section{INTRODUCTION}

Alcohol use disorder (AUD) is a common mental disorder that results in substantial disease burden worldwide, including in China (Yang et al. 2013). The presence of comorbid mental disorders in individuals with AUD has been associated with a lower quality of life, poorer clinical outcomes, and increased risk of suicidal behavior (Andrews et al. 2002; Bolton et al.

\footnotetext{
* Address for correspondence: M. R. Phillips, 3210 Humin Rd., Shanghai 201108, China.

(Email: mphillipschina@outlook.com)

t These authors contributed equally to the work.
}

2010), so understanding the pattern of AUD co-morbidity can help clinicians and service agencies provide more targeted and, thus, more effective treatments for AUD. Comorbidity data are also needed to adjust the estimates of AUD-related disease burden reported in the Global Burden of Disease studies.

Most studies from high-income countries report a moderate-to-strong positive association between AUD and other mental disorders (Andrews et al. 2002; Kessler et al. 2005; Hasin et al. 2007; FlensborgMadsen et al. 2009; Markon, 2010; Chou et al. 2012).

- The strongest association is between AUD and drug use disorder (DUD). Individuals with AUD are

This is an Open Access article, distributed under the terms of the Creative Commons Attribution licence (http://creative commons.org/licenses/by/4.0/), which permits unrestricted re-use, distribution, and reproduction in any medium, provided the original work is properly cited. 
10-to-20 times more likely to have comorbid DUD than individuals without AUD (Farrell, 2001; Andrews et al. 2002; Kessler et al. 2005; Hasin et al. 2007; Flensborg-Madsen et al. 2009; Markon, 2010; Chou et al. 2012).

- Cross-sectional studies have shown that individuals with AUD are 3-8 times more likely to have mood or anxiety disorders (Farrell, 2001; Andrews et al. 2002; Kessler et al. 2005; Hasin et al. 2007; Flensborg-Madsen et al. 2009; Markon, 2010; Chou et al. 2012); and these findings are supported by prospective studies (Fergusson et al. 2009; Boden \& Fergusson, 2011). Proposed mechanisms for this relationship include (1) neurophysiological changes induced by extended exposure to alcohol, which increase the risk of developing mood and anxiety disorders, and (2) using alcohol to alleviate mood or anxiety symptoms (i.e., the 'self-medication theory') (Bolton et al. 2009; McCarty et al. 2012).

- Limited evidence based on responses to screening questions from a few population-based studies suggests positive associations between AUD and disorders characterized by severe cognitive impairment, such as schizophrenia, dementia, and mental retardation (Degenhardt et al. 2001; McMillan et al. 2009; Lopes et al. 2010).

AUD is not rare in Chinese males. A previous meta-analysis found a similar prevalence of AUD in Chinese males compared with males in high-income western countries (Cheng et al. 2015). However, drinking patterns in China are quite different from western drinking patterns. The most common drinking pattern in China is heavy drinking episodes and mutual intoxication among male business associates or friends with the goal of building and enhancing important social relationship; heavy drinking at college parties is relatively rare (Cochrane et al. 2003). So it is not surprising that the demographic pattern of AUD identified in China is quite different from that reported in highincome countries. For example, there is a much higher male-to-female ratio of AUD $(30: 1)$ and a much later mean age of onset of AUD (30 years of age) than reported in high-income countries (Cheng et al. 2015; Phillips et al. 2017). In addition, married Chinese males are more likely to have AUD than single Chinese males, and employed Chinese males are more likely to have AUD than unemployed Chinese males (Cheng et al. 2015; Phillips et al. 2017). Given these differences and the importance of comorbidity patterns in the prevention and management of AUD, it is important to determine whether or not the strong positive association between AUD and other mental disorders reported in high-income countries also exists in China. In a previous paper (Phillips et al. 2017) we reported an inverse association between AUD and the presence of any non-AUD mental disorder diagnosed according to criteria specified in the 4th edition of the American Psychiatric Association Diagnostic and Statistical Manual of Mental Disorders (DSM-IV); that is, men with AUD were less likely to have another mental disorder than men without AUD.

In the current paper we elaborate on this surprising finding among men in China by using data from five large community-based psychiatric epidemiological studies conducted from 2002 to 2012 to address the following questions:

- What is the association between AUD and specific types of non-AUD mental disorders?

- Are there demographic differences between individuals with AUD who do and do not have comorbid mental disorders?

\section{METHODS}

\section{Sample}

Data used for this study come from five large population-based surveys: three province-wide surveys in Qinghai, Shandong, and Zhejiang; a survey in the largest prefecture of Gansu Province (Tianshui Prefecture); and a survey in Tianjin - one of the large municipalities in China that are administratively equivalent to a Province. The Zhejiang survey was conducted in 2001, the Tianjin survey was conducted in 2012, and the other three surveys were conducted in 2005. All five surveys used the same protocol. The sampling frame for these surveys accounted for $>12 \%$ of the adult Chinese population. A multi-stage stratified sampling method yielded a representative sample of non-institutionalized adults living in these five locations. In brief, the initial stratification classified prefectures by socioeconomic characteristics; the probability proportionate to size (PPS) method was used to select primary sampling units (i.e., neighborhoods in urban areas and villages in rural areas); and simple random sampling was used to identify individuals from each primary sampling unit to be approached to participate in the survey. Ninety-four percent of individuals who were selected participated in the survey (74752 out of 79177). All participants provided informed consent and the study was approved by local institutional review boards.

\section{Assessments of mental disorders and demographic variables}

A two-stage interview procedure was employed. First, an expanded version of the General Health Questionnaire (Goldberg \& Williams, 1988) was used 
to classify each participant's risk of having a mental disorder. In the second stage, trained psychiatrists administered the validated Chinese version of the Structured Clinical Interview for DSM-IV-TR axis I disorders (SCID) (Phillips \& Liu, 2011) to all high-risk individuals and to a random sample of those classified in the first stage as moderate or low risk of having a mental disorder. The final diagnosis was based on criteria specified in DSM-IV (American Psychiatric Association, 2000). The differential selection for administering this diagnostic exam was accounted for by applying probability weights in the analysis. This method identified 22140 individuals who were selected for the second-stage diagnostic interview, among whom 21015 (94\%) accepted the interview. Both the General Health Questionnaire and SCID have been validated in the Chinese population (Zhou et al. 1997; Yang et al. 2003). More details about the sampling process and analysis weights are provided in previous publications (Phillips et al. 2009, 2017) and in the Supplementary Material for this paper.

The DSM-IV diagnosis of AUD includes two subcategories - alcohol dependence and nondependent alcohol abuse. Individuals who manifest at least one of four socially maladaptive drinking behaviors (shown in Table 3) within a 12-month period have 'alcohol abuse' and those in whom three or more of the seven features of dependence (shown in Table 3) occur within a 12-month period have 'alcohol dependence'; but alcohol dependence is prioritized over alcohol abuse, so individuals who simultaneously meet both criteria are given the single diagnosis of alcohol dependence. In this study AUD status was categorized into three mutually exclusive groups:

(1) 'never had AUD,' those who never met criteria of alcohol abuse or alcohol dependence;

(2) 'current AUD,' those who met criteria of alcohol abuse or alcohol dependence at any time during the 30 days prior to the assessment, and

(3) 'past AUD,' those who previously met criteria of abuse or dependence but did not meet abuse or dependence criteria at any time during the 30 days prior to the assessment.

Due to the small number of AUD cases among females $(n=48)$, the final analytical sample was limited to the 9619 males who completed the SCID interview. Each of the seven clinical features of alcohol dependence was coded by the interviewing psychiatrist following standard SCID skip patterns. The four clinical features of alcohol abuse were coded for the 8744 men who did not meet lifetime criteria of alcohol dependence.

Seven categories of non-AUD mental disorders were considered:
- mood disorders including major depressive disorder, dysthymia, bipolar disorder, mood disorder due to physical conditions, and mood disorders not otherwise specified (NOS);

- anxiety disorders including panic disorder, agoraphobia without panic, social phobia, specific phobias, obsessive compulsive disorder, post-traumatic stress disorder, generalized anxiety disorder, anxiety disorder due to physical conditions, and anxiety disorder NOS;

- psychotic disorders including schizophrenia, schizophreniform disorder, schizoaffective disorder, delusional disorder, brief psychotic disorder, psychotic disorder due to physical conditions, psychotic disorder due to substance use, and psychotic disorder NOS;

- drug use disorders including DSM-IV abuse and dependence of internationally regulated drugs;

- somatoform disorders including somatization disorder, conversion disorder, pain disorder, hypochondriasis, body dysmorphic disorder, and undifferentiated somatoform disorder; and

- mental retardation, and dementia (not assessed in Zhejiang Province).

Substance-induced mood disorder and substanceinduced anxiety disorder were not included in the combined categories of non-AUD mood disorder and non-AUD anxiety disorder due to their causal relationship with substance use (mainly alcohol use). More details about the assessment of mental disorders are available elsewhere (Phillips et al. 2009, 2017). Individuals who met the DSM-IV criteria for non-AUD mental disorders at any time in the month prior to the assessment were considered cases of 'current disorders'.

Demographic variables recorded included age, residency (urban or rural), marital status, years of education, household income (quartiles based on provincespecific income levels), and employment status based on self-reported information. Duration of AUD was calculated by subtracting the age of onset of AUD from the age at the time of the assessment.

\section{Statistical methods}

We conducted three sets of analyses. (1) The 30-day prevalence of non-AUD mental disorders was estimated for each of the three AUD groups (i.e., never, current, and past AUD). Associations between AUD and non-AUD mental disorders were estimated using logistic regression models that adjusted for age. Associations were estimated both for diagnostic categories (e.g., all mood disorders and all anxiety disorders) and for specific types of mental disorders. When zero cell counts occurred, a design-based F-test was used to provide $p$ values to gauge the statistical 
significance of differences. (2) Socio-demographic characteristics were compared between three groups of respondents: those with 'current AUD with current non-AUD mental disorders', 'current AUD without current non-AUD mental disorders', and 'no current AUD'. (3) We compared the occurrence of each of the seven clinical features of alcohol dependence between individuals with current AUD with and without current non-AUD mental disorders. We also compared the occurrence of each of the four features of alcohol abuse between individuals with current AUD without lifetime alcohol dependence (i.e., those with 'nondependent alcohol abuse') with and without current non-AUD mental disorders. Generalized estimation equations (GEE) were used to assess heterogeneity of estimates across clinical features and to provide robust estimates while taking into account the correlation between different AUD clinical features (Liang \& Zeger, 1986). First, a specific-slope model with product terms for each clinical feature is estimated; if specificslope estimates for all clinical features are similar (i.e., $p$ values for all product terms are greater than $0.10)$, a more parsimonious common-slope model that provides one summary estimate for all clinical features is estimated (Liang \& Zeger, 1986).

For the first two sets of analyses, we first generated site-specific estimates for the five sites and then summarized the estimates using meta-analysis (we consider this method superior to the traditional datapooling method because it takes into account crossprovince heterogeneity - which our previous reports found to be substantial (Phillips et al. 2009, 2017)). A random-effect estimator was used when there is indication of heterogeneity (i.e., $I^{2}>50 \%$ and the $p$ value for heterogeneity test $<0.05)$. For the third set of analyses, we pooled data from all five sites because the low occurrence of some clinical features in each site made it impractical to conduct meta-analyses.

Probability weights were used to take into account differential selection across risk levels and poststratification factors. The Taylor Series Linearization method was used to take into account design effects, such as possible clustering within primary sampling units. The precision of estimates was reported using $95 \%$ confidence intervals.

\section{RESULTS}

\section{Prevalence of other mental disorders in persons with and without current or past AUD}

Table 1 shows the adjusted current (i.e., 30-day) prevalence of mood disorders, anxiety disorders, psychotic disorders, and other non-AUD disorders for the three AUD strata. The meta-analysis summaries of odds ratios show statistically significant inverse associations between current AUD and the combined categories of any current mood disorder and any current anxiety disorder. With the exception of bipolar disorder, all specific mood disorders considered are less prevalent in individuals with current AUD than in individuals who had never had AUD, though the differences were not statistically significant. Among the six specific types of anxiety disorders considered, anxiety disorders NOS was significantly more prevalent among individuals who had never had AUD than in individuals with current AUD $(2.2 \%$ v. $1.6 \%)$. The age-adjusted prevalence of DUD and mental retardation were also significantly lower among individuals with current AUD compared with those who had never had AUD. There was also a borderline inverse association between current AUD and 'other psychotic disorders' (OR 0.3, $p=0.054$ ).

There were no statistically significant differences in the current prevalence of non-AUD mental disorders between persons who had never had AUD and those with a prior history of AUD. Post-hoc comparisons of the prevalence of current non-AUD diagnoses between individuals with past AUD v. that in individuals with current AUD found that individuals with past AUD had a higher prevalence of current mood disorder NOS (OR 1.5; 95\% CI 1.1-2.1) and current anxiety disorder NOS (OR 3.5, 95\% CI 1.4-8.9), and a statistical trend for a higher prevalence of current dysthymia (OR 2.1, $p=0.069$ ).

Table 2 shows estimates for the prevalence of current non-AUD diagnoses among individuals with current and past alcohol dependence and current and past nondependent alcohol abuse (i.e., alcohol abuse in those without lifetime alcohol dependence). The results for current alcohol dependence and current nondependent alcohol abuse are generally congruent to those for the combined current AUD category shown in Table 1. No consistent differences were observed in the prevalence of any of the current non-AUD diagnoses between individuals with past dependence and past nondependent abuse. However, individuals with past nondependent alcohol abuse were more likely than those with current nondependent alcohol abuse to have any current mood disorder (OR 1.8, 95\% CI 1.1-2.8).

\section{Comparison of men with no current AUD, current AUD without current non-AUD mental disorders, and AUD with current non-AUD mental disorders}

The mean (S.D.) age of onset of AUD symptoms in men with current AUD was similar in those with and without current non-AUD mental disorders (28.6 years $v$. 30.0 years, $t=1.25, p=0.213$ ). Similarly, the duration of illness in men with current AUD was similar in 
Table 1. Current prevalence of DSM-IV mental disorders among adult males from five provinces in China stratified by current and past alcohol use disorder (AUD) status, 2001-2012

\begin{tabular}{|c|c|c|c|c|c|c|c|c|}
\hline \multirow[b]{2}{*}{ Type of mental disorder } & \multicolumn{2}{|c|}{$\begin{array}{l}\text { Never had AUD } \\
(n=7664)\end{array}$} & \multicolumn{3}{|c|}{$\begin{array}{l}\text { Current AUD } \\
(n=1571)\end{array}$} & \multicolumn{3}{|c|}{$\begin{array}{l}\text { Past AUD } \\
(n=384)\end{array}$} \\
\hline & $n^{\mathrm{a}}$ & $\%^{\mathrm{b}}$ & $n^{\mathrm{a}}$ & $\%$ & $\mathrm{OR}^{\mathrm{c}}(95 \% \mathrm{CI})$ & $n^{\mathrm{a}}$ & $\%$ b & $\mathrm{OR}^{\mathrm{c}}(95 \% \mathrm{CI})$ \\
\hline Any current mood disorder & 1064 & 4.4 & 61 & 3.3 & $0.6(0.4-0.8)$ & 30 & 4.1 & $0.8(0.4-1.4)$ \\
\hline Bipolar disorders & 45 & 0.3 & 7 & 0.4 & $2.3(0.8-6.8)$ & 1 & 0.6 & $0.6(0.1-6.1)$ \\
\hline Major depressive disorder & 381 & 1.3 & 26 & 1.3 & $0.8(0.5-1.6)$ & 16 & 1.3 & $0.9(0.5-1.7)$ \\
\hline Dysthymia & 308 & 1.3 & 15 & 1.1 & $0.5(0.3-1.1)$ & 7 & 1.6 & $1.2(0.4-3.8)$ \\
\hline Illness-induced mood disorder & 44 & 0.1 & 0 & 0.0 & $p=0.247^{\mathrm{d}}$ & 2 & 1.3 & $p=0.954^{\mathrm{d}}$ \\
\hline Mood disorders NOS & 358 & 1.6 & 16 & 1.2 & $0.6(0.2-2.4)$ & 9 & 2.9 & $2.3(0.5-11.2)$ \\
\hline Any current anxiety disorder & 739 & 3.4 & 49 & 2.2 & $0.5(0.4-0.8)$ & 29 & 5.9 & $1.3(0.8-2.2)$ \\
\hline Panic disorders and phobias & 68 & 0.3 & 3 & 0.3 & $0.5(0.1-1.5)$ & 5 & 0.7 & $1.7(0.6-5.3)$ \\
\hline Obsessive-compulsive disorder & 17 & 0.1 & 0 & 0 & $p=0.272^{\mathrm{d}}$ & 0 & 0 & $p=0.578^{\mathrm{d}}$ \\
\hline Post-traumatic stress disorder & 24 & 0.1 & 1 & 0.1 & $0.3(<0.1-2.8)$ & 1 & 0.1 & $1.6(0.2-14.5)$ \\
\hline Generalized anxiety disorder & 183 & 0.9 & 9 & 0.5 & $0.8(0.3-1.8)$ & 2 & 0.6 & $1.1(0.1-13.0)$ \\
\hline Illness-induced anxiety disorder & 29 & 0.2 & 2 & 0.3 & $2.3(0.3-19.0)$ & 1 & 0.4 & $2.8(0.3-22.8)$ \\
\hline Anxiety disorders NOS & 456 & 2.2 & 38 & 1.6 & $0.6(0.4-1.0)$ & 21 & 3.5 & $1.3(0.6-2.8)$ \\
\hline Any current psychotic disorder & 211 & 1.0 & 3 & 0.5 & $0.4(0.1-2.0)$ & 4 & 0.6 & $0.5(0.2-1.5)$ \\
\hline Schizophrenia & 181 & 0.8 & 3 & 0.5 & $0.5(0.1-2.2)$ & 3 & 0.4 & $0.5(0.1-2.6)$ \\
\hline Other psychotic disorders & 39 & 0.2 & 2 & 0.1 & $0.3(0.1-1.0)$ & 1 & 1.7 & $3.9(0.5-28.2)$ \\
\hline \multicolumn{9}{|l|}{ Other current non-AUD disorders } \\
\hline Drug use disorders & 26 & 0.1 & 0 & 0.0 & $p=0.029^{\mathrm{d}}$ & 3 & 0.3 & $2.2(0.5-10.9)$ \\
\hline Somatoform disorders ${ }^{\mathrm{e}}$ & 35 & 0.2 & 1 & 0.3 & $8.5(0.5-157.2)$ & 1 & 0.4 & $0.8(0.1-7.6)$ \\
\hline Mental retardation ${ }^{\mathrm{f}}$ & 114 & 0.9 & 0 & 0.0 & $p=0.022^{\mathrm{d}}$ & 2 & 0.4 & $0.5(0.1-3.1)$ \\
\hline Dementia $^{\mathrm{f}}$ & 88 & 0.2 & 1 & 0.1 & $0.4(<0.1-3.3)$ & 8 & 0.5 & $1.1(0.4-2.6)$ \\
\hline
\end{tabular}

DSM-IV, fourth edition of the diagnostic and statistical manual of mental disorders; NOS, not otherwise specified.

${ }^{\text {a }}$ Number of cases.

${ }^{\mathrm{b}}$ Weighted percentage adjusted for sampling, clustering, and post-stratified to the sampling frame.

${ }^{c}$ Odds ratio and its $95 \%$ confidence interval adjusting for age; reference group for each OR are individuals who never had AUD. Bold font indicates statistical significance at 0.05 level.

${ }^{\mathrm{d}} p$ value of designed-based $F$-test (reference = individuals who never had AUD).

e Includes somatization disorder, conversion disorder, pain disorder, hypochondriasis, body dysmorphic disorder, and undifferentiated somatoform disorder.

${ }^{\mathrm{f}}$ Mental retardation and dementia were not assessed in Zhejiang province.

those with and without current non-AUD mental disorders (14.1 years $v .15 .2$ years, $t=-0.55, p=0.586$ ).

Table 3 shows the results of comparing the demographic characteristics of the three groups of men. Several interesting associations were identified.

- AGE: compared with men without current AUD, men with current AUD (with or without current non-AUD mental disorders) are more likely to be middle-aged adults (i.e., 40-54 years of age) than young adults (i.e., 18-39 years of age).

- RESIDENCE: men with both current AUD and current non-AUD mental disorders are more likely to reside in urban areas compared with men in the other two groups.

- MARITAL STATUS: men with current AUD without non-AUD mental disorders are more likely to be currently married than men in the other two groups.

- LEVEL OF EDUCATION: men with both current AUD and current non-AUD mental disorders are much less likely to have 10 years or more of schooling compared to men without current AUD.

- FAMILY INCOME LEVEL: the family income of men with current AUD without current non-AUD mental disorders is more likely to be in the top two quartiles of family income (i.e., higher) than the family income of men without current AUD; and the family income of men with both current AUD and current non-AUD mental disorders is more likely to be in the lowest quartile of family income than the family income of men in the other two groups.

- EMPLOYMENT STATUS: men with current AUD without current non-AUD mental disorders are 
Table 2. Current prevalence of non-AUD mental disorders among adult males from five provinces in China stratified by current and past alcohol dependence and by current and past nondependent alcohol abuse (i.e., alcohol abuse in those without current or past alcohol dependence), 2001-2012

\begin{tabular}{|c|c|c|c|c|c|c|c|c|c|c|c|c|c|c|}
\hline \multirow{2}{*}{$\begin{array}{l}\text { Categories of mental disorders } \\
\text { (current diagnosis only) }\end{array}$} & \multicolumn{2}{|c|}{$\begin{array}{l}\text { Never had } \\
\text { AUD } \\
(n=7664)\end{array}$} & \multicolumn{3}{|c|}{$\begin{array}{l}\text { Current nondependent abuse } \\
(n=690)\end{array}$} & \multicolumn{3}{|c|}{$\begin{array}{l}\text { Current dependence } \\
(n=881)\end{array}$} & \multicolumn{3}{|c|}{$\begin{array}{l}\text { Past nondependent abuse } \\
(n=294)\end{array}$} & \multicolumn{3}{|c|}{$\begin{array}{l}\text { Past dependence } \\
(n=90)\end{array}$} \\
\hline & $n^{\mathrm{a}}$ & $\%^{\mathrm{b}}$ & $n^{\mathrm{a}}$ & $\%{ }^{\mathrm{b}}$ & $\mathrm{OR}^{\mathrm{c}}(95 \% \mathrm{CI})$ & $n^{\mathrm{a}}$ & $\%^{\mathrm{b}}$ & $\mathrm{OR}^{\mathrm{c}}(95 \% \mathrm{CI})$ & $n^{\mathrm{a}}$ & $\%^{\mathrm{b}}$ & $\mathrm{OR}^{\mathrm{c}}(95 \% \mathrm{CI})$ & $n^{\mathrm{a}}$ & $\%^{\mathrm{b}}$ & $\mathrm{OR}^{\mathrm{c}}(95 \% \mathrm{CI})$ \\
\hline Any current mood disorder & 1064 & 4.4 & 27 & 3.4 & $0.7(0.4-1.1)$ & 34 & 3.1 & $0.5(0.3-0.8)$ & 21 & 5.1 & $1.2(0.4-3.9)$ & 9 & 2.8 & $0.5(0.2-1.2)$ \\
\hline Bipolar disorders & 45 & 0.3 & 4 & 0.7 & $4.1(1.3-13.2)$ & 3 & 0.2 & $1.3(0.3-5.4)$ & 1 & 0.8 & $0.7(0.1-7.9)$ & 0 & 0 & $p=0.813^{\mathrm{d}}$ \\
\hline Major depressive disorder & 381 & 1.3 & 8 & 0.5 & $0.5(0.2-1.1)$ & 18 & 1.9 & $1.2(0.5-2.7)$ & 10 & 1.3 & $1.0(0.4-2.2)$ & 6 & 1.9 & $1.5(0.5-4.4)$ \\
\hline Dysthymia & 308 & 1.3 & 3 & 1.8 & $1.5(0.5-5.2)$ & 12 & 1.1 & $0.4(0.2-0.97)$ & 4 & 2.3 & $1.9(0.2-17.4)$ & 3 & 1.0 & $1.0(0.1-6.7)$ \\
\hline Mood disorders NOS & 358 & 1.6 & 12 & 1.6 & $1.0(0.3-3.5)$ & 4 & 0.7 & $0.3(0.1-1.2)$ & 7 & 3.9 & $2.9(0.5-16.3)$ & 2 & 0.7 & $0.4(0.1-1.9)$ \\
\hline Illness-induced mood disorder & 44 & 0.1 & 0 & 0 & $p=0.337^{\mathrm{d}}$ & 0 & 0 & $p=0.462^{\mathrm{d}}$ & 2 & 1.5 & $p=0.827^{\mathrm{d}}$ & 0 & 0 & $p=0.792^{\mathrm{d}}$ \\
\hline Any current anxiety disorder & 739 & 3.4 & 14 & 1.2 & $0.3(0.2-0.7)$ & 35 & 3.6 & $0.7(0.5-1.2)$ & 23 & 5.2 & $1.3(0.6-2.5)$ & 6 & 5.9 & $1.3(0.3-5.6)$ \\
\hline Panic disorders and phobias & 68 & 0.3 & 1 & 0.2 & $0.2(<0.1-1.6)$ & 2 & 1.0 & $1.4(0.1-20.9)$ & 4 & 0.5 & $1.6(0.6-4.6)$ & 1 & 5.0 & $p=0.02^{\mathrm{d}}$ \\
\hline Obsessive-compulsive disorder & 17 & 0.1 & 0 & 0 & $p=0.402^{\mathrm{d}}$ & 0 & 0 & $p=0.493^{\mathrm{d}}$ & 0 & 0 & $p=0.613^{\mathrm{d}}$ & 0 & 0 & $p=0.818^{\mathrm{d}}$ \\
\hline Post-traumatic stress disorder & 24 & 0.1 & 0 & 0 & $p=0.378^{\mathrm{d}}$ & 1 & 0.1 & $0.4(0.1-3.5)$ & 1 & 0.1 & $2.2(0.2-21.0)$ & 0 & 0 & $p=0.798^{\mathrm{d}}$ \\
\hline Generalized anxiety disorder & 183 & 0.9 & 4 & 0.4 & $0.8(0.2-3.8)$ & 5 & 0.5 & $1.0(0.2-2.2)$ & 2 & 0.7 & $1.3(0.1-13.9)$ & 0 & 0 & $p=0.586^{\mathrm{d}}$ \\
\hline Illness-induced anxiety disorder & 29 & 0.2 & 2 & 0.7 & $7.8(0.97-60.5)$ & 0 & 0 & $p=0.398^{\mathrm{d}}$ & 0 & 0 & $P>0.999^{\mathrm{d}}$ & 1 & 0.9 & $6.4(0.7-56.1)$ \\
\hline Anxiety disorders NOS & 456 & 2.2 & 10 & 1.1 & $0.5(0.2-1.3)$ & 28 & 2.6 & $0.8(0.4-1.3)$ & 16 & 3.8 & $1.5(0.6-3.5)$ & 5 & 5.2 & $1.0(0.3-3.6)$ \\
\hline Any current psychotic disorder & 211 & 1.0 & 1 & 1.1 & $0.8(0.1-6.4)$ & 2 & 0.2 & $0.2(<0.1-1.03)$ & 3 & 0.7 & $0.5(0.1-1.9)$ & 1 & 2.1 & $2.2(0.3-17.3)$ \\
\hline Schizophrenia & 181 & 0.8 & 1 & 1.1 & $0.9(0.1-6.8)$ & 2 & 0.2 & $0.2(<0.1-1.1)$ & 2 & 0.4 & $0.4(0.1-2.0)$ & 1 & 2.1 & $2.3(0.3-18.2)$ \\
\hline Other psychotic disorders & 39 & 0.2 & 0 & 0 & $p=0.312^{\mathrm{d}}$ & 2 & 0.5 & $2.9(0.6-13.8)$ & 1 & 2.0 & $4.6(0.6-34.5)$ & 0 & 0 & $p=0.761^{\mathrm{d}}$ \\
\hline \multicolumn{15}{|l|}{ Other current non-AUD disorders } \\
\hline Drug use disorders & 26 & 0.1 & 0 & 0 & $p=0.313^{\mathrm{d}}$ & 0 & 0 & $p=0.397^{\mathrm{d}}$ & 3 & 0.3 & $2.7(0.5-13.4)$ & 0 & 0 & $p=0.776^{\mathrm{d}}$ \\
\hline Somatoform disorders ${ }^{\mathrm{e}}$ & 35 & 0.2 & 0 & 0 & $p=0.409^{\mathrm{d}}$ & 1 & 0.3 & $11.8(0.5-260.1)$ & 1 & 0.4 & $1.1(0.1-9.7)$ & 0 & 0 & $p=0.801^{\mathrm{d}}$ \\
\hline Mental retardation ${ }^{\mathrm{d}, \mathrm{f}}$ & 114 & 0.9 & 0 & 0 & $p=0.118^{\mathrm{d}}$ & 0 & 0 & $p=0.278^{\mathrm{d}}$ & 1 & 0.2 & $0.2(<0.1-2.0)$ & 1 & 2.1 & $3.6(0.4-31.3)$ \\
\hline Dementia $^{f}$ & 87 & 0.2 & 0 & 0 & $p=0.091^{\mathrm{d}}$ & 1 & 0.1 & $0.5(0.1-4.3)$ & 5 & 0.4 & $0.9(0.3-2.8)$ & 3 & 0.9 & $2.1(0.6-8.0)$ \\
\hline
\end{tabular}

AUD, alcohol use disorder; DSM-IV, Fourth edition of the diagnostic and statistical manual of mental disorders; NOS, not otherwise specified.

${ }^{\text {a }}$ Number of cases.

${ }^{\mathrm{b}}$ Weighted percentage adjusted for sampling, clustering, and post-stratified to the sampling frame.

${ }^{c}$ Odds ratio adjusting for age. Reference group who never had AUD. Bold font indicates statistical significance at 0.05 level.

${ }^{\mathrm{d}} p$ value of designed-based $F$-test (reference = individuals who never had AUD).

e Somatoform disorders included somatization disorder, conversion disorder, pain disorder, hypochondriasis, body dysmorphic disorder, and undifferentiated somatoform disorder.

${ }^{\mathrm{f}}$ Mental retardation and dementia were not assessed in Zhejiang province. 
Table 3. Comparison of selected socio-demographic characteristics of adult males from five provinces in China stratified by current status of alcohol use disorder (AUD) and comorbid non-AUD mental disorders, 2001-2012

\begin{tabular}{|c|c|c|c|c|c|c|c|c|c|c|}
\hline \multirow[b]{2}{*}{ Variables } & \multirow[b]{2}{*}{ Categories } & \multicolumn{2}{|c|}{$\begin{array}{l}\text { No current } \\
\text { AUD } \\
(n=8048)\end{array}$} & \multicolumn{3}{|c|}{$\begin{array}{l}\text { Current AUD without current } \\
\text { non-AUD mental disorders } \\
(n=1468)\end{array}$} & \multicolumn{4}{|c|}{$\begin{array}{l}\text { Current AUD with current } \\
\text { non-AUD mental disorders } \\
(n=103)\end{array}$} \\
\hline & & $n$ & $\%^{\mathrm{b}}$ & $n$ & $\%^{b}$ & $\mathrm{OR}^{\mathrm{c}, \mathrm{d}}(95 \% \mathrm{CI})$ & $n$ & $\%^{\mathrm{b}}$ & $\mathrm{OR}^{\mathrm{c}, \mathrm{d}}(95 \% \mathrm{CI})$ & $\mathrm{OR}^{\mathrm{c}, \mathrm{e}}(95 \% \mathrm{CI})$ \\
\hline \multirow[t]{3}{*}{ Age groups } & $18-39$ & 2706 & 55.3 & 409 & 45.1 & & 27 & 28.9 & & \\
\hline & $40-54$ & 2542 & 25.1 & 661 & 37.3 & $1.6(1.2-2.1)$ & 53 & 54.9 & $3.7(2.2-6.2)$ & $1.7(0.9-3.2)$ \\
\hline & $55+$ & 2797 & 19.6 & 401 & 17.6 & $1.2(0.8-1.7)$ & 23 & 16.3 & $1.0(0.4-2.2)$ & $0.4(0.2-1.1)$ \\
\hline \multirow[t]{2}{*}{ Residence } & Urban & 2990 & 38.3 & 504 & 29.3 & & 45 & 47.1 & & \\
\hline & Rural & 5128 & 61.7 & 967 & 70.7 & $0.9(0.6-1.2)$ & 58 & 52.9 & $0.1(0.1-0.3)$ & $0.2(0.1-0.6)$ \\
\hline \multirow[t]{3}{*}{ Marital status (six missing cases) } & Currently married/cohabitant & 6335 & 76.5 & 1308 & 89.7 & & 80 & 84.3 & & \\
\hline & Never married & 648 & 6.9 & 88 & 3.9 & $0.5(0.4-0.7)$ & 13 & 9.1 & $0.9(0.4-1.9)$ & $2.5(1.1-6.3)$ \\
\hline & Separated/divorced/widowed & 1128 & 16.6 & 75 & 6.4 & $0.3(0.2-0.4)$ & 10 & 6.6 & $0.7(0.4-1.5)$ & $2.3(1.0-5.3)$ \\
\hline \multirow[t]{3}{*}{ Years of schooling (four missing cases) } & $0-6$ & 3340 & 30.2 & 576 & 37.5 & & 38 & 30.6 & & \\
\hline & $7-9$ & 2742 & 37.4 & 570 & 37.9 & $0.9(0.7-1.1)$ & 38 & 44.2 & $0.9(0.4-1.9)$ & $1.2(0.6-2.7)$ \\
\hline & $10+$ & 2032 & 32.4 & 325 & 24.6 & $0.8(0.6-1.1)$ & 27 & 25.3 & $0.1(<0.1-0.2)$ & $0.5(0.2-1.1)$ \\
\hline \multirow[t]{4}{*}{ Household income levels ${ }^{\mathrm{f}}$ (eight missing cases) } & Lowest quartile & 2313 & 23.6 & 343 & 23.8 & & 31 & 32.9 & & \\
\hline & Second quartile & 1884 & 22.3 & 347 & 21.8 & $1.0(0.8-1.4)$ & 22 & 14.0 & $0.3(0.1-0.6)$ & $0.3(0.1-0.6)$ \\
\hline & Third quartile & 1981 & 26.1 & 373 & 25.7 & $1.4(1.0-1.9)$ & 25 & 30.6 & $3.1(1.6-5.8)$ & $2.0(0.9-4.3)$ \\
\hline & Highest quartile & 1918 & 28.0 & 405 & 28.7 & $1.6(1.2-2.3)$ & 23 & 22.4 & $0.7(0.2-1.7)$ & $0.5(0.2-1.3)$ \\
\hline \multirow[t]{6}{*}{ Employment (71 missing cases) } & Farmer & 3676 & 42.7 & 731 & 57.3 & & 35 & 25.5 & & \\
\hline & Factory worker & 736 & 12.9 & 192 & 10.2 & $0.8(0.6-1.3)$ & 13 & 20.3 & $2.4(0.8-7.1)$ & $3.1(0.9-10.3)$ \\
\hline & Professionals & 144 & 13.3 & 143 & 10.8 & $0.9(0.6-1.4)$ & 11 & 17.4 & $2.3(0.7-7.3)$ & $2.6(0.7-9.3)$ \\
\hline & Self-employed & 1010 & 17.7 & 195 & 14.6 & $0.7(0.5-1.1)$ & 16 & 10.9 & $1.4(0.6-3.5)$ & $1.9(0.7-5.0)$ \\
\hline & Retired & 1155 & 7.5 & 145 & 4.4 & $0.4(0.3-0.7)$ & 12 & 9.9 & $1.8(0.9-3.7)$ & $2.0(0.7-5.3)$ \\
\hline & Unemployed & 563 & 5.9 & 60 & 2.7 & $0.7(0.4-1.3)$ & 15 & 16.0 & $5.1(2.1-12.5)$ & $6.3(2.1-18.6)$ \\
\hline
\end{tabular}

${ }^{\text {a }}$ The mean (S.E.) duration of AUD in 1476 persons without concurrent mental disorders was 14.2 (0.7) years and the corresponding value in 98 persons with concurrent AUD and other mental disorders was 14.8 (2.0) years; the duration of AUD was not statistically different between the two groups (OR 0.99, 95\% CI 0.96-1.03).

${ }^{\mathrm{b}}$ Column percentages adjusted for sampling, clustering and post-stratified to the sampling frame. May not add up to exactly $100 \%$ due to rounding.

${ }^{c}$ Meta-analytic summary of province-specific odds ratios adjusted for age category, province of residence, urban $v$. rural residence, marital status, level of education, family income, and work status using a random-effect estimator. Bold font indicates statistical significance at 0.05 level.

$\mathrm{d}$ The comparison group is 'no current AUD'.

e The comparison group is 'current AUD without current non-AUD mental disorders'.

${ }^{\mathrm{f}}$ Province-specific quartiles of income. 
less likely to be retired than men without current AUD; and men with current comorbid AUD and current non-AUD mental disorders are much more likely to be unemployed than men in the other two groups.

In summary, compared with men without current AUD, men with current AUD in the absence of other non-AUD mental disorders are more likely to be middle-aged, to be currently married, and to have higher family incomes. On the other hand, compared with men without current AUD, men with both current AUD and current non-AUD mental disorders are less likely to have finished high school and more likely to be middle-aged, to live in urban areas, to have lower family incomes, and to be unemployed. And compared with men with current AUD without non-AUD mental disorders, those with both current AUD and current non-AUD mental disorders are less likely to be currently married and more likely to live in urban areas, to have low family incomes, and to be unemployed.

\section{Prevalence of clinical features of AUD in individuals with current AUD with and without current non-AUD mental disorders}

Table 4 compares the prevalence of the different DSM-IV symptoms of alcohol dependence between men with current AUD with and without current non-AUD mental disorders and, separately, the prevalence of the symptoms of alcohol abuse between men with current AUD without current or past alcohol dependence (i.e., 'nondependent alcohol abuse') with and without current non-AUD mental disorders.

The seven features of alcohol dependence were assessed in all 1574 respondents with current AUD. The age-adjusted GEE model revealed no robust variations in the association of the symptoms and the presence of current non-AUD mental disorders in men with current AUD between the seven clinical features ( $p>0.1$ for all product terms), so we used the commonslope model as a summary measure for the seven features. Compared with men who had current AUD without current non-AUD mental disorders, those with both current AUD and current non-AUD mental disorders are more likely to experience clinical features of alcohol dependence (OR from the common-slope model is $1.6,95 \%$ CI 1.1-2.6).

The four socially maladaptive behaviors that are the diagnostic symptoms of alcohol abuse were assessed in 692 men with AUD who did not have current or past alcohol dependence - that is, in men with nondependent alcohol abuse. The prevalence of role interference was significantly higher in men with current nondependent alcohol abuse without current non-AUD mental disorders than in men with both current nondependent alcohol abuse and current non-AUD mental disorders. There was no statistically significant difference in the prevalence of the other three symptoms of alcohol abuse between the two groups, though this may partly be due to the relatively small number of individuals with both current nondependent alcohol abuse and current non-AUD mental disorders $(n=38)$. The age-adjusted GEE model found that the association for legal problems is different from that for role interference $(p<0.05$ for the product term), so it was not possible to use a common-slope model as a summary measure for the four symptoms.

\section{DISCUSSION}

\section{Main findings}

In contrast to a large body of literature from other countries indicating moderate to strong positive correlations between AUD and non-AUD mental disorders (Andrews et al. 2002; Kessler et al. 2005; Hasin et al. 2007; Flensborg-Madsen et al. 2009; Markon, 2010; Chou et al. 2012), this large, representative study from China found that men with AUD were significantly less likely to have other mental disorders than men who do not have AUD. The demographic correlates of AUD were also different from those reported elsewhere: compared with men without AUD, men with AUD in the absence of other mental disorders were more likely to be middle-aged, to be currently married, and to have higher family incomes. However, men with both AUD and other mental disorders were less likely than men without AUD to have finished high school and more likely to live in urban areas, to have lower family incomes, and to be unemployed. Compared with men with current AUD without other current mental disorders, those with both current AUD and other mental disorders were more likely to manifest the clinical features of alcohol dependence.

\section{Limitations and strengths}

Several important study limitations merit attention. First, given the low prevalence of AUD in females and the low prevalence of some specific mental disorders, the analysis was necessarily restricted to males and it was not possible to separately report results for some specific mental disorders. Second, this study is cross-sectional so the temporal relationships of AUD and non-AUD mental disorders are unknown; the onset of comorbid non-AUD mental disorders may have occurred before, after or simultaneously with the onset of AUD. Third, the study combines data collected over a 11-year period (from 2001 to 2012), so it may not reflect changes in the relationship 
Table 4. Comparison of the occurrence of clinical features of alcohol use disorder (AUD) among adult males from five provinces of China with current AUD who do or do not have current non-AUD mental disorders, 2001-2012

\begin{tabular}{|c|c|c|c|c|c|c|}
\hline & \multicolumn{2}{|c|}{$\begin{array}{l}\text { Current AUD } \\
\text { without current } \\
\text { non-AUD mental } \\
\text { disorder }(n=1468)\end{array}$} & \multicolumn{4}{|c|}{$\begin{array}{l}\text { Current AUD with current non-AUD mental } \\
\text { disorder }(n=103)\end{array}$} \\
\hline & $n^{\mathrm{a}}$ & $\%^{\mathrm{b}}$ & $n^{\mathrm{a}}$ & $\%^{\mathrm{b}}$ & $\mathrm{OR}^{\mathrm{c}}$ & $95 \% \mathrm{CI}$ \\
\hline Meets lifetime alcohol dependence criteria & 814 & 39.9 & 65 & 60.6 & 2.2 & $1.1-4.5$ \\
\hline \multicolumn{7}{|l|}{ Clinical features of alcohol dependence } \\
\hline Often drinks more than planned to & 855 & 46.2 & 68 & 59.1 & 1.4 & $0.7-3.0$ \\
\hline Failed attempts to cut down drinking & 575 & 29.7 & 59 & 51.9 & 2.3 & $1.0-4.9$ \\
\hline Spends lots of time drinking & 582 & 27.4 & 42 & 30.1 & 1.0 & $0.5-2.0$ \\
\hline Gives up activities because of drinking & 359 & 16.2 & 29 & 21.4 & 1.3 & $0.7-2.7$ \\
\hline Continues to drink despite health problems & 477 & 21.8 & 39 & 36.6 & 1.9 & $1.0-3.7$ \\
\hline Tolerance, increases intake for same effect & 611 & 30.1 & 56 & 42.9 & 1.5 & $0.7-3.4$ \\
\hline Withdrawal symptoms when abstinent & 476 & 22.8 & 38 & 31.8 & 1.4 & $0.6-3.2$ \\
\hline \multirow[t]{6}{*}{ Clinical features of alcohol abuse } & \multirow{5}{*}{\multicolumn{2}{|c|}{$\begin{array}{l}\text { Current } \\
\text { nondependent } \\
\text { alcohol abuse }^{\mathrm{d}} \\
\text { without current } \\
\text { non-AUD mental } \\
\text { disorder }(n=654)\end{array}$}} & \multirow{5}{*}{\multicolumn{4}{|c|}{$\begin{array}{l}\text { Current nondependent alcohol abuse }{ }^{\mathrm{d}} \text { with } \\
\text { current non-AUD mental disorder }(n=38)^{\mathrm{d}}\end{array}$}} \\
\hline & & & & & & \\
\hline & & & & & & \\
\hline & & & & & & \\
\hline & & & & & & \\
\hline & $n^{\mathrm{a}}$ & $\%^{b}$ & $n^{\mathrm{a}}$ & $\%^{\mathrm{b}}$ & $\mathrm{OR}^{\mathrm{c}}$ & $95 \% \mathrm{CI}$ \\
\hline Role interference & 517 & 79.2 & 29 & 56.2 & 0.3 & $0.1-0.9$ \\
\hline Social problems & 227 & 23.9 & 13 & 30.6 & 1.3 & $0.5-3.7$ \\
\hline Legal problems & 27 & 3.0 & 4 & 6.9 & 2.3 & $0.5-10.6$ \\
\hline Hazardous drinking & 380 & 49.6 & 25 & 56.5 & 1.3 & $0.5-3.5$ \\
\hline
\end{tabular}

\footnotetext{
${ }^{a}$ Number of cases.

${ }^{b}$ Weighted lifetime occurrence (\%) of AUD-related outcomes in each group. Column percentages adjusted for sampling, clustering and post-stratified to the sampling frame.

${ }^{\mathrm{c}}$ Odds ratio and $95 \%$ confidence interval of having the symptom (or disorder) adjusting for age compared with the prevalence in men with current AUD who did not have a current mood or anxiety disorder.

${ }^{\mathrm{d}}$ Current AUD in men with no current or past alcohol dependence.
}

between AUD and non-AUD mental disorders over time. Fourth, tobacco dependence and DSM-IV Axis II mental disorders (i.e., personality disorders) were not considered in the study. Fifth, under-reporting of past symptoms of mental disorders is well documented in the psychiatric epidemiology literature, especially for mild or moderate symptoms from the distant past (Engels et al. 1997; Streiner et al. 2009); to minimize the effect of under-reporting on our conclusions, our analysis focuses on the current status of AUD and non-AUD mental disorders. Finally, the diagnostic instrument used in the study (SCID) follows the diagnostic hierarchy of DSM-IV, which does not assess the presence of the clinical features of current or past alcohol abuse in individuals with current or past alcohol dependence, so it was only possible to assess the pattern of clinical features of alcohol abuse among men with non-dependent alcohol abuse.

Despite these limitations, the study has good internal and external validity. It reports on the largest representative sample of community-dwelling individuals in China for whom both AUD and non-AUD mental disorders were systematically assessed by trained psychiatrists. There was a very high response rate and the source population was clearly defined, so the population to which the results can be generalized is well characterized. And we used up-to-date statistical methods to adjust for a variety of design effects and reported overall results based on meta-analysis of results at each of the five study sites 
(rather than pooling results across all five sites), steps that reduce potential biases in the estimates.

\section{Interpretation}

We conclude that our main results are robust, that is, the substantial differences identified between AUD in China and elsewhere are real, not the result of methodological or sampling limitations. A previous prospective study about the relationship of alcohol use and depressive symptoms in China (Cheng et al. 2016) supports our finding of an inverse relationship between AUD and mental disorders: it found that current drinking predicted lower rates of subsequent depressive symptoms and current depressive symptoms predicted lower rates of subsequent drinking among middle-aged and elderly Chinese.

What could explain the differences between AUD in China to that reported elsewhere? Both environmental and genetic factors play a role. In contemporary China, drinking is most commonly used to celebrate or to enhance relationships with friends, coworkers, supervisors, and business partners (primarily among men); solo drinking to relieve stress is quite uncommon. Thus the primary function of alcohol in China is to enhance social status. This different cultural role of alcohol compared with that in the West is associated with differences both in the demographic profile and in the comorbidity pattern of AUD: men who drink regularly in China have higher social functioning and status and they are less likely to have comorbid mental disorders than men who do not drink regularly. However, the higher social functioning is only true for Chinese men with AUD without a comorbid mental disorder (Phillips et al. 2017); the relatively small group of AUD cases with other comorbid mental disorders are more likely to be unemployed, to have low income, and to be divorced - a pattern which is consistent with the demographic profile of all individuals with AUD reported in western countries (Pirkola et al. 2005; Hasin et al. 2007).

Genetic characteristics of Chinese of Han ethnicity (the vast majority of Chinese citizens) may also play a role in the different relationship between AUD and 'internalizing' mental disorders (e.g., mood and anxiety disorders) between Chinese and western populations. Several theories of addiction suggest the association between AUD and mood disorders occurs because individuals with mood problems drink alcohol for its anxiolytic effects and escalate their drinking via negative reinforcement mechanisms, which then exacerbate their mood or anxiety symptoms during abstinence (Koob \& Kreek, 2007). However, a large proportion of Han Chinese do not metabolize ethanol as efficiently as most individuals living in western countries due to the differential distribution of $\mathrm{ADH}$ and ALDH gene polymorphisms (Thomasson et al. 1991; Goedde et al. 1992). This has been hypothesized as a protective factor that limits heavy drinking in Chinese because individuals with the polymorphisms may stop drinking when they experience the unpleasant flushing effects that occurs due to the accumulation of acetaldehyde. It is possible that the undesired flushing effects in Chinese with the polymorphisms prevent them from feeling the anxiolytic effects of alcohol, making it less likely that they would use alcohol to 'treat' their psychological symptoms. If true, this would eliminate the positive association between AUD and mood and anxiety disorders. A previous study in primary care settings in 14 countries (including China) identified an 'internalization' latent construct that gives rise to anxiety, depression, and somatization but is unrelated to problematic alcohol use (Krueger et al. 2003), which supports our suggestion that the 'internalizing' process is not the strongest driving force for alcohol use problems in China. Other supportive evidence includes a previous study in the USA documenting largely null associations between substance use disorders and depression or anxiety disorders in Asian Americans, against a background of positive associations in other American ethnic groups (Wu et al. 2013). Future studies with genotype assessment will help elucidate these possibilities.

Due to the lack of information on other 'externalizing' disorders, such as anti-social personality disorder and conduct disorder, we were not able to analyze the comorbidity pattern between AUD and externalizing disorders. However, there were very few cases of nonalcohol DUD in our sample $(n=29)$ and only three of them had a history of AUD, a pattern that is very different from that reported in the West (Hasin et al. 2007). We expect that this is partly due to the very low accessibility to illegal drugs in China (i.e., an environmental factor), a situation that may change as China continues to open to the West.

Our results also touch on the issue of the value of combining alcohol abuse and alcohol dependence into a single disorder - AUD. When comparing the clinical profiles of men with current AUD only and men with both current AUD and another mental disorder, the seven clinical features of alcohol dependence were homogeneous: they were all more likely to occur in men with comorbid AUD and other mental disorders than in men who only had AUD. However, the four socially maladaptive behaviors characteristic of alcohol abuse were heterogeneous: role interference was more common in the AUD only group and legal problems were more common in the AUD and other mental disorder group. Given the small number of cases with nondependent AUD and other mental 
disorders $(n=38)$ and the exclusion of individuals with prior alcohol dependence from the analysis of the symptoms of alcohol abuse, this result needs to be confirmed by further research, but this finding is in line with the conceptualization of the 'alcohol dependence syndrome' (Edwards, 1986). It also suggests the need to be cautious when combining the symptoms of alcohol dependence and socially maladaptive drinking behavior into a single-dimension AUD category, as has been done in the revised DSM-5 classification (Edwards, 2012; Hasin et al. 2013).

\section{Conclusion}

As is true for other complex behavioral conditions, the AUD phenotype, the demographic profile of AUD, and the association of AUD with other mental and physical disorders are the result of the interaction of cultural, social and genetic factors. Cross-cultural differences in these factors underlie the substantial differences in the demographic characteristics and comorbidity pattern of AUD between China and western countries. Understanding the origin of these differences both helps to elaborate the general theory of addictive behaviors and helps identify appropriate methods and outcome measures when developing community-specific or cohort-specific interventions to prevent or treat addictive disorders.

\section{Supplementary material}

The supplementary material for this article can be found at https://doi.org/10.1017/S0033291717001337

\section{Acknowledgements}

We thank the participating institutions, clinicians, and individuals in Shandong, Zhejiang, Tianjin, Qinghai, and Gansu for their active support and our funding agencies The survey that provided data for the current analysis was done under the auspices of the Small Grants Program to improve the Quality and Implementation of Mental Health Research in China, which was coordinated by Xue Zhang at Peking Union Medical College and MRP at Beijing Hui Long Guan Hospital, and supported by the China Medical Board of New York (grant number 02-777). The Zhejiang survey was part of the WHO and Chinese Ministry of Health Mental Health Project supported by a WHO grant (GL/GLO/MNH /343/XE/00.J.999.00). The Shandong Provincial Bureau of Health also provided additional support for the survey in Shandong. The separate 2011-2012 survey in the Tianjin Municipality was supported by the Tianjin Municipal Bureau of Finance. The analysis was supported by the China Medical Board of New York (grant number 13-165).

\section{Ethical standards}

The authors assert that all procedures contributing to this work comply with the ethical standards of the relevant national and institutional committees on human experimentation and with the Helsinki Declaration of 1975, as revised in 2008.

\section{Declaration of interest}

None.

\section{References}

American Psychiatric Association (2000). Diagnostic and Statistical Manual of Mental Disorders., 4th edn TR.

Andrews G, Slade T, Issakidis C (2002). Deconstructing current comorbidity: data from the Australian National Survey of Mental Health and Well-Being. British Journal of Psychiatry 181, 306-314.

Boden JM, Fergusson DM (2011). Alcohol and depression. Addiction 106, 906-914.

Bolton JM, Pagura J, Enns MW, Grant B, Sareen J (2010). A population-based longitudinal study of risk factors for suicide attempts in major depressive disorder. Journal of Psychiatric Research 44, 817-826.

Bolton JM, Robinson J, Sareen J (2009). Self-medication of mood disorders with alcohol and drugs in the National Epidemiologic Survey on Alcohol and Related Conditions. Journal of Affective Disorders 115, 367-375.

Cheng HG, Chen S, McBride O, Phillips MR (2016). Prospective relationship of depressive symptoms, drinking, and tobacco smoking among middle-aged and elderly community-dwelling adults: results from the China Health and Retirement Longitudinal Study (CHARLS). Journal of Affective Disorders 195, 136-143.

Cheng HG, Deng F, Xiong W, Phillips MR (2015). Prevalence of alcohol use disorders in mainland China: a systematic review. Addiction 110, 761-774.

Chou SP, Lee HK, Cho MJ, Park JI, Dawson DA, Grant BF (2012). Alcohol use disorders, nicotine dependence, and co-occurring mood and anxiety disorders in the United States and South Korea-a cross-national comparison. Alcoholism: Clinical and Experimental Research 36, 654-662.

Cochrane J, Chen H, Conigrave KM, Hao W (2003). Alcohol use in China. Alcohol and Alcoholism 38, 537-542.

Degenhardt L, Hall W, Lynskey M (2001). Alcohol, cannabis and tobacco use among Australians: a comparison of their associations with other drug use and use disorders, affective and anxiety disorders, and psychosis. Addiction 96, 1603-1614+1689.

Edwards G (1986). The alcohol dependence syndrome: a concept as stimulus to enquiry. British Journal of Addiction 81, 171-183.

Edwards G (2012). 'The evil genius of the habit': DSM-5 seen in historical context. Journal of Studies on Alcohol and Drugs 73, 699-701. 
Engels RCME, Knibbe RA, Drop MJ (1997). Inconsistencies in adolescents' self-reports of initiation of alcohol and tobacco use. Addictive Behaviors 22, 613-623.

Farrell M (2001). Nicotine, alcohol and drug dependence and psychiatric comorbidity: results of a national household survey. British Journal of Psychiatry 179, 432-437.

Fergusson DM, Boden JM, Horwood LJ (2009). Tests of causal links between alcohol abuse or dependence and major depression. Archives of General Psychiatry 66, 260-266.

Flensborg-Madsen T, Mortensen EL, Knop J, Becker U, Sher L, Gronbaek M (2009). Comorbidity and temporal ordering of alcohol use disorders and other psychiatric disorders: results from a Danish register-based study. Comprehensive Psychiatry 50, 307-314.

Goedde HW, Agarwal DP, Fritze G, Meier-Tackmann D, Singh S, Beckmann G, Bhatia K, Chen LZ, Fang B, Lisker R, Paik YK, Rothhammer F, Saha N, Segal B, Srivastava LM, Czeizel A (1992). Distribution of ADH2 and ALDH2 genotypes in different populations. Human Genetics 88, 344-346.

Goldberg P, Williams P (1988). A User's Guide to the GHQ. NFER-Nelson: Windsor, UK.

Hasin DS, O'Brien CP, Auriacombe M, Borges G, Bucholz K, Budney A, Compton WM, Crowley T, Ling W, Petry NM, Schuckit M, Grant BF (2013). DSM-5 criteria for substance use disorders: recommendations and rationale. American Journal of Psychiatry 170, 834-851.

Hasin DS, Stinson FS, Ogburn E, Grant BF (2007). Prevalence, correlates, disability, and comorbidity of DSM-IV alcohol abuse and dependence in the United States: results from the National Epidemiologic Survey on Alcohol and Related Conditions. Archives of General Psychiatry 64, 830-842.

Kessler RC, Chiu WT, Demler O, Walters EE, Merikangas KR, Walters EE (2005). Prevalence, severity, and comorbidity of 12-month DSM-IV disorders in the National Comorbidity Survey Replication. Archives of General Psychiatry 62, 617-627.

Koob G, Kreek MJ (2007). Stress, dysregulation of drug reward pathways, and the transition to drug dependence. American Journal of Psychiatry 164, 1149-1159.

Krueger RF, Chentsova-Dutton YE, Markon KE, Goldberg D, Ormel J (2003). A cross-cultural study of the structure of comorbidity among common psychopathological syndromes in the general health care setting. Journal of Abnormal Psychology 112, 437-447.

Liang K-Y, Zeger SL (1986). Longitudinal data analysis using generalized linear models. Biometrika 73, 13-22.

Lopes MA, Furtado EF, Ferrioli E, Litvoc J, De Campos Bottino CM (2010). Prevalence of alcohol-related problems in an elderly population and their association with cognitive impairment and dementia. Alcoholism: Clinical and Experimental Research 34, 726-733.

Markon KE (2010). Modeling psychopathology structure: a symptom-level analysis of Axis I and II disorders. Psychological Medicine 40, 273-288.

McCarty CA, Wymbs BT, King KM, Mason WA, Vander Stoep A, McCauley E, Baer J (2012). Developmental consistency in associations between depressive symptoms and alcohol use in early adolescence. Journal of Studies on Alcohol and Drugs 73, 444-453.

McMillan KA, Enns MW, Cox BJ, Sareen J (2009).

Comorbidity of Axis I and II Mental Disorders with Schizophrenia and Psychotic Disorders: findings from the National Epidemiologic Survey on Alcohol and Related Conditions. Canadian Journal of Psychiatry-Revue Canadienne De Psychiatrie 54, 477-486.

Phillips MR, Cheng HG, Li X, Zhang J, Shi Q, Xu G, Song Z, Ding Z, Pang S (2017). Prevalence, correlates, comorbidity, and age of onset of alcohol use disorders in adult males from five provinces in China. Drug and Alcohol Dependence 173, 170-177.

Phillips MR, Liu X (2011). In Translated and adapted Chinese version of Structured Clinical Interview for DSM-IV-TR Axis I Disorders, Research Version, Patient Edition (SCID-I/P) (eds MB First, RL Spitzer, M Gibbon, JBW Williams), Suicide Research and Prevention Center, Shanghai Mental Health Center: Shanghai.

Phillips MR, Zhang J, Shi Q, Song Z, Ding Z, Pang S, Li X, Zhang Y, Wang Z (2009). Prevalence, treatment, and associated disability of mental disorders in four provinces in China during 2001-05: an epidemiological survey. Lancet 373, 2041-2053.

Pirkola SP, Isometsä E, Suvisaari J, Aro H, Joukamaa M, Poikolainen K, Koskinen S, Aromaa A, Lönnqvist JK (2005). DSM-IV mood-, anxiety- and alcohol use disorders and their comorbidity in the Finnish general population. Results from the Health 2000 Study. Social Psychiatry and Psychiatric Epidemiology 40, 1-10.

Streiner DL, Patten SB, Anthony JC, Cairney J (2009). Has 'lifetime prevalence' reached the end of its life? An examination of the concept. International Journal of Methods in Psychiatric Research 18, 221-228.

Thomasson HR, Edenberg HJ, Crabb DW, Mai XL, Jerome RE, Li TK, Wang SP, Lin YT, Lu RB, Yin SJ (1991). Alcohol and aldehyde dehydrogenase genotypes and alcoholism in Chinese men. American Journal of Human Genetics 48, 677681.

Wu LT, Blazer DG, Gersing KR, Burchett B, Swartz MS, Mannelli P (2013). Comorbid substance use disorders with other Axis I and II mental disorders among treatment-seeking Asian Americans, Native Hawaiians/ Pacific Islanders, and mixed-race people. Journal of Psychiatric Research 47, 1940-1948.

Yang G, Wang Y, Zeng Y, Gao GF, Liang X, Zhou M, Wan X Yu S, Jiang Y, Naghavi M, Vos T, Wang H, Lopez AD, Murray CJL (2013). Rapid health transition in China, 1990-2010: findings from the Global Burden of Disease Study 2010. Lancet 381, 1987-2015.

Yang T, Huang L, Wu Z (2003). The application of Chinese health questionnaire for mental disorder screening in community settings in mainland China. Zhonghua Liu Xing Bing Xue Za Zhi 24, 769-773.

Zhou RY, Zhang YH, Peng B, Lie XHZC (1997). Comparison of three diagnostic criteria for the diagnosis of schizophrenia and mood disorders. Chinese Journal of Psychiatry 1, 45-49. 